\title{
Mood State and Human Evaluation of the Thermal Environment Using Virtual Settings
}

\author{
Anwar Ibrahim ${ }^{1,}$, Hikmat Ali $^{1}$, Aroub Zghoul ${ }^{1}$, Suha Jaradat ${ }^{2}$ \\ ${ }^{1}$ College of Architecture and Design, Department of Architecture, Jordan University of \\ Science and Technology, Jordan. \\ 2 School of Engineering and The Built Environment, Edinburgh Napier University, \\ Edinburgh, UK \\ *Corresponding Author. Email: afibrahim@just.edu.jo
}

\begin{abstract}
Many complaints about indoor conditions are related to unsatisfactory thermal environments. Most research on Thermal Comfort (TC) considered physical parameters for settings and users yet marginalized the influence of user's psychological aspects in the process of thermal sensation. Immersive Virtual Reality (VR) has been used in the built environment to simulate real scenarios. This research examines the effect of mood states on human evaluation of the thermal environment in virtual settings. Forty-four university students from Jordan participated. The experiment followed the "Experimental Design Method" using thermally controlled chamber and TC evaluation using psychological responses developed by Fanger. The participants completed the PANAS-X pre-mood test before watching a video that targeted eliciting predetermined mood states; anger and happiness. The participants were then immersed in two virtual environments and asked to complete ASHRAE 7- point scale of TC. General Linear model was used to analyse the data. The results revealed a relationship between TC, mood state and quality of the indoor environment. Humans' judgment on TC is a variable mental reaction. The research presents differences between the evaluation of angry and happy people to their thermal environments. This study expands research on the indoor environment quality and develops TC evaluation strategies.
\end{abstract}

Keywords

Mood state, Human evaluation, Thermal sensation, Virtual settings, Indoor environment quality 


\section{Introduction}

We spend most of our time indoor in artificial thermal environments which affect human beings. Properly designed indoor environments are essential for pleasant living and optimized task performance in the work place. Amongst other parameters, Thermal Comfort (TC) level is one of the scales that measure human`s solace in physical settings. It indicates the thermal balance of the body as determined by the operative temperature and moderated by environmental and personal parameters. However, providing the standard thermal comfort operative temperature in an indoor environment does not necessarily imply an all-inclusive users' thermal satisfaction. Differentiations in individuals' physiology and psychology lead to a state of inconsistencies in humans' reactions to thermal environments. ${ }^{1}$ Thermal sensation was described as a person-specific sensory experience and a psychological phenomenon that is mainly influenced by personal aspects. $^{2}$

Studies on TC are classified within the domain of the physical and technical sciences. Three major trends of TC studies were identified: positivistic and reductionist approach, multivariate and systems-oriented approach and applied and conservationoriented approach. ${ }^{3}$ The former is a physical and laboratory-based trend which exported set of standards, indexes and bioclimatic charts in indoor and outdoor thermal conditions. Olgyay $^{4}$ was the first to chart the comfort zone in architecture domain. His Bio-Climatic Chart defined the boundaries of under heated, comfortable and overheated zones of outdoor thermal conditions based on a dry bulb temperature and humidity level; then he added air velocity factor to extend these boundaries. ${ }^{4}$ This chart was then developed to new versions adopted from ASHRAE standards by considering physiological and physical variables such as clothing level and directional radiation effect. ${ }^{5}$ For indoor thermal condition, a thermal index was developed based on a steady-state heat balance between the 
body and the surrounded environment. ${ }^{6}$ By the time, several experimental studies outlined some problems related to those models in terms of application and temperature preferences. For example, these models are not applicable in extreme cold and hot conditions (e.g. hot arid climates). Most importantly, a large gap was found between the perceived temperature and the predicted temperature by these models. ${ }^{7,8}$

The second trend assumes a more psychological and multivariate approach. ${ }^{9}$ It clarifies that the physical approaches are not comprehensive and need to be adjusted by taking into account additional variables such as gender differences, adaptation time, geographical location and extreme conditions. ${ }^{10}$ This approach considers two axes: TC's subjective parameters, including individual differences, and the subjective evaluation technique (semantic differential scales) which human beings used to assist their thermal satisfaction. ${ }^{3}$ Psychological and subjective variables, especially the individual's mental state, are central sources for individual variations in thermal sensation. ${ }^{7,11,12}$ Another trend is an applied oriented research that focuses on issues of energy conservation such as enhancing occupant behaviour and obtaining thermally comfortable spaces. ${ }^{13}$ Recent studies also focused on evaluating the local thermal comfort to enhance the design and selection of heating systems using a thermal manikin with human thermoregulatory control. ${ }^{14}$ This control, which was used in the manikin, imitates human thermoregulation and interacts with the environment using real-time measurements.

\section{Mental state and emotion}

Mental state is defined as a psychological outcome response to objective and subjective indicators. ${ }^{15}$ Emotion is a subjective output of the mental state, and is defined as a mental response resulted from the interconnection between the brain and the physiological actions of the body. ${ }^{16}$ When developing attitudes towards the surrounding 
environment, emotions are initiated and act as a mediator component in the undergoing perception process, thus they play a substantial role in generating diverse responses. ${ }^{17}$

The relationship between mood state and its direct influence on environmental perception can be best understood through the Gestalt theory. ${ }^{18}$ According to this theory, the mind and the humans’ psychological actions are in control of their perception. ${ }^{19}$ However, the mood, as a mental state, may moderate these psychological processes which in turn sway perception. Based on the Gestalt theory, Smith and Lazarus ${ }^{20}$ argue that although the initial 'perception activity’ precedes an emotional response in a flow of psychological events, the successive perception will be influenced by the beforehand generated emotions. The human evaluation may incorporate any of the environment's numerous attributes as well as the various amount of emotions or mental activities. ${ }^{20}$

Appraisal theories state that human`s judgment and prediction for the surroundings is partially mediated and judged by their emotions. ${ }^{21}$ Therefore, researchers should pay extra attention to the occupants' psychological processing and evaluation rather than focusing on the influence of environmental interior attributes on occupant behaviour. ${ }^{19}$ Likewise, Keltner et al. ${ }^{21}$ highlighted the role of mood state as a subjective controller to our perception of the outside circumstances and people. Similarly a model was developed by utilizing mood as an assessment tool to measure the effect of extreme surrounding conditions. $^{22}$ The model stipulates that the perception of environmental features is highly subjective and strongly bonded to personal factors, including mood response. ${ }^{22}$

On the other hand, a model of a man-environment relationship, see Figure 1, clarified that the process of evaluating the environment is processed through the 'Mediator Variables' (including perception, cognition, and effective process); in which the mood state is one of the effective processes. ${ }^{23}$ Therefore, the mood state acts as a filtration layer 
to the humans' perception of the physical setting and may strongly impact their effective response, thus shaping their preferences and expectations. ${ }^{23}$ A number of experimental studies addressed the spatial characteristics of spaces and their relationship with mood triggering. For example, examining the colour-mood relations in physical settings proved that warm colours tended to stimulate positive arousal responses while cool colours tended to evoke calmer and peaceful emotions. ${ }^{24-27}$ Another study demonstrated that naturally-lit environment decreases the positive mood and negatively impacts the task performance. ${ }^{28}$ The effect of lighting environment on the work performance in office buildings was examined to identify the relationship between lighting illuminance, uniformity of illuminance, correlated colour temperature as well as the productivity of workers using subjective questionnaires, objective physiological tests and computerized neurobehavioural tests. ${ }^{29}$ This experiment highlighted the significance of designing bespoke illumination environment for each work place. However, those studies did not consider humans`perception processing of physical setting indicators; whereas the perception action of our minds precedes the mood response itself. ${ }^{19}$ 


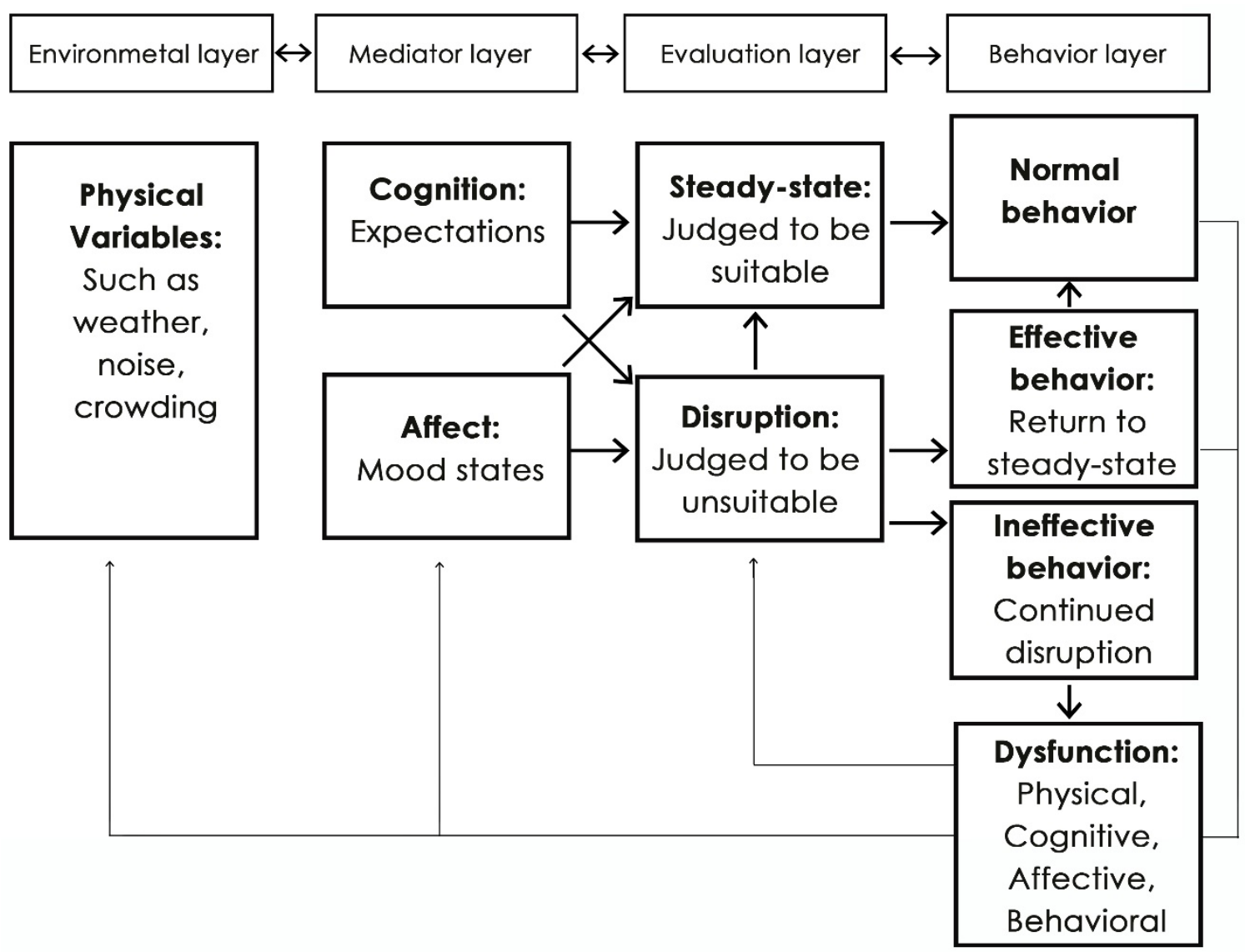

Figure 1. A model to understand the man-environment relationship. ${ }^{23}$

\section{Human mood, thermal conditions and task performance}

The human mood and its relationship with thermal conditions as well as its effect on task performance gained special attention on experimental research. For example, Muller et al. ${ }^{30}$ demonstrated that the negative mood states are altered during cold thermal conditions; therefore, the human cognition, daily activities and wellbeing are disrupted. Psychologically, the thermal conditions can stimulate and provoke the brain to invoke various responses and behaviours such as motivating the creativity level and influencing social interaction. ${ }^{30}$ Muller et al. ${ }^{30}$ added that the human mood could be modified by increasing the temperature which in return would improve the human`s cognition and perception. In addition, cold environments tend to increase the negative mood states' 
effect and worsen people's thermal sensation. ${ }^{31}$ Hot conditions could also relate a negative effect on mood state and task performance.

Temperature as an environmental factor plays an important role in human`s perception. ${ }^{32}$ For example, Batra and Garg ${ }^{32}$ indicated that higher degrees of temperature led to slower understanding and perceiving of information; and poor retention of the task as compared to the moderate level of temperature. A study on military personnel concluded that exercise during hot conditions led to mood deterioration and reduction in cognitive performance, whereas soldiers' thermal sensation was the highest. ${ }^{33}$ Although physiological responses to thermal conditions are more predictable, the impact of these conditions on human`s thermal perception varies over one’s psychological state. For example, performing a given task in cold environment will induce greater oxygen consumption compared to performing the same task in a thermally neutral condition. ${ }^{34}$

Personality traits may also mitigate the influence of the surrounding thermal conditions. Some personalities intuitively possess the ability to control their mood state regardless of the surrounding thermal condition, in order to complete their activities successfully. ${ }^{22}$ However, other personalities do not have such ability and will have their mood control both their perception and performance. ${ }^{22}$ Moreover from psychological perspective, the ambient temperature is possibly mediated through human`s mood. ${ }^{35}$ Zohng and Leonardelli ${ }^{36}$ argued that emotions can also evoke thermal responses. They debated that negative feelings (e.g. social exclusion) induced cold sensation while positive feeling (e.g. feeling loved) induced warmth sensation, regardless the environment temperature. ${ }^{36}$

Immersive Virtual Reality (VR) hardware and software have been developed and become available to professionals, business, consumers and researchers. Users wear VR headsets or navigate a 3D environment using joysticks and keyboards. VR technology and systems have been widely used in many fields including gaming and human-computer 
interactions; ${ }^{37}$ body space and human social interactions; ${ }^{38,39}$ gaming and health-related domains; ${ }^{40}$ training for emergency evacuation in buildings; ${ }^{41}$ and many more VR applications in the built environment. VR offers a wide range of opportunities but also has limitations. ${ }^{39}$ For example, experiments highlighted a difference in skin temperature and thermal sensation in the immersive VR environment and real indoor environment. Researchers developed various methods to decrease the difference between VR and the real environment and enable users to move freely in the virtual environment to create a natural VR experience. ${ }^{42}$

As a conclusion, TC is a crucial concern in indoor quality assessment research. People working or living in uncomfortably hot or cold environments are prone to performing unsafe behaviours as their abilities of decision-making and/or performing manual tasks are altered. Previous research on TC and the developed models paid attention to the environment and user's physical factors and relegated the psychological aspects of the user. Although earlier studies have proved the strong influence of mood state on the way people evaluate and judge their environments, the influence of mood state on the thermal perception of the environment has not been thoroughly examined yet. This research attempts to bridge this gap by examining how thermal sensation might be altered by the interaction of the emotional states, the operative temperature and environmental design attributes. By doing so, this study expands research on the indoor environment quality and develops the TC evaluation strategies through involving the subjective psychological parameters. In other words, this research provides a more realistic description about human's thermal sensation and the perception of the quality of the indoor environment. The aim of this research is to find ways in which humans' thermal sensation of the indoor environment might be affected by the mood state. The objectives associated with the overall aim of the study are: 
1- To examine the effect of the positive and negative mood states on humans' perception and evaluation of their thermal comfort under high, low and neutral thermal conditions in two different virtual settings.

2- To identify the related design attributes that potentially monitor the effect of mood state on the thermal comfort perception.

\section{Methodology}

The research methodology depends on experimental design method (Factorial Design) in addition to self-report measurement to answer the questionnaire. It enables the experimenter to study the joint effect of independent factors (design parameters) on a response (dependent factor) ${ }^{43}$. This experiment is a $3 \times 3 \times 2$ factorial design; (mood state, operative temperature, virtual setting). The mood state factor has 3 levels (high, medium and low), the operative temperature has also 3 levels $\left(15^{\circ} \mathrm{C}, 21^{\circ} \mathrm{C}\right.$, and $\left.27^{\circ} \mathrm{C}\right)$. Finally, the virtual setting has two levels (environment 1, environment 2). The main analytical tools for this design are; the main effect of each level for each factor which clarifies the mean response values, and the interaction effect which clarifies the mean response values of all factors together at all possible combinations of their settings, see Figure 2. 


\section{Virtual setting}

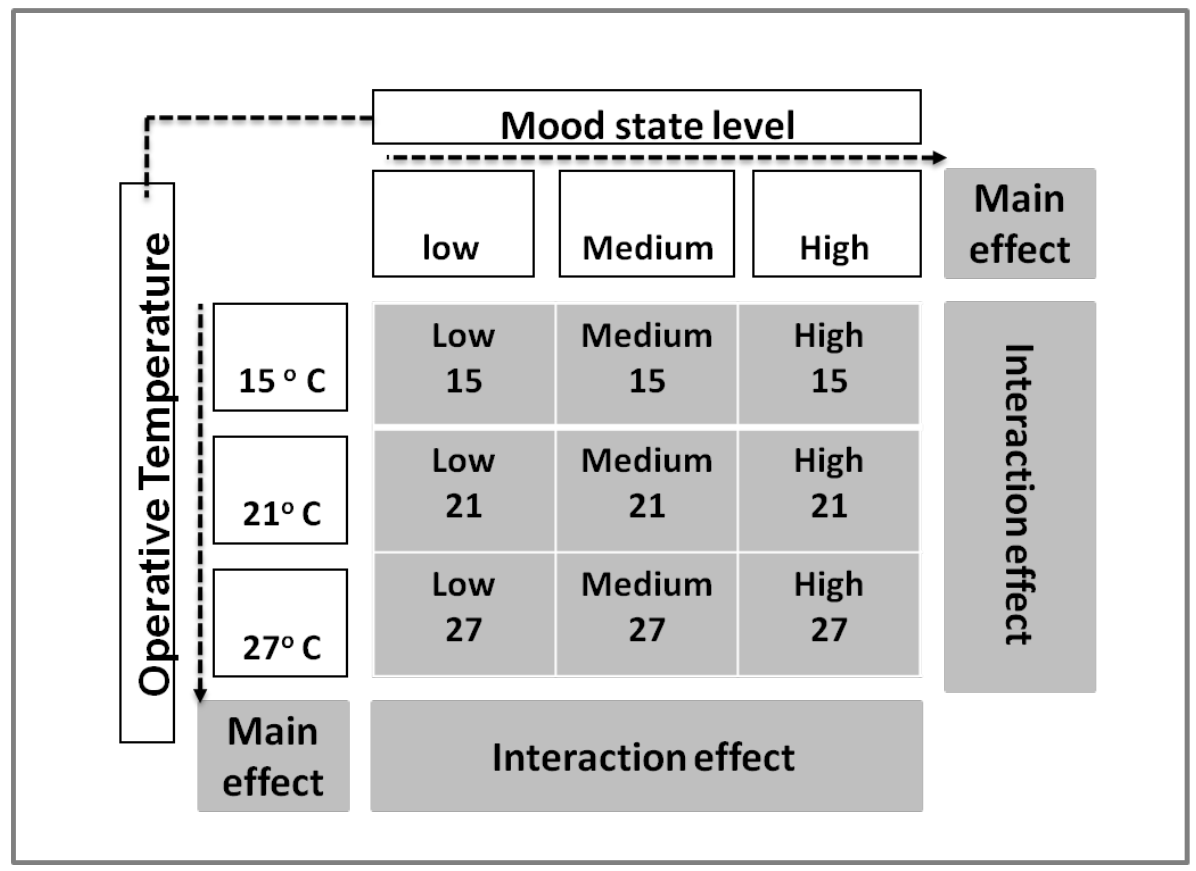

Figure 2. The factorial design for tested models

Forty-four (44) university students from Jordan University of Science and Technology with age range of (18 - 22) participated in this experiment. Participants were from different majors and consisted of twenty-two (22) male students and twenty-two (22) female students. Each participant was to experience three thermal scenarios with a gap of one week, see Table 1 . Some physical attributes were fixed including: clothing insulation level with a range from 0.4 - 0.6 CLO and the humidity level with a range from $30 \%-50 \%$ depending on the investigated thermal level. The experiment was held in November with almost similar outside weather conditions. These selected operative temperatures reflected the average seasonal temperatures in Jordan (winter, spring and summer respectively). 
Table 1. Main characterstics of the participants.

\begin{tabular}{l|l}
\hline \multicolumn{1}{c|}{ Properety } & \multicolumn{1}{c}{ Note } \\
\hline Participants Number & 44 \\
Clo level & $0.4-0.6$ \\
Participants weight $(\mathrm{kg})$ & $50-60$ female \\
& $60-70$ male \\
Participants majors & Engineering and science \\
Participants ages & $18-22$ \\
Humidity rate range & $30 \%-50 \%$ \\
\hline
\end{tabular}

\section{Experimental setting}

The experiment was conducted in a Lab at the Department of Architecture. The Lab was divided into two zones, the first one is a transitional space and the second is internal thermally controlled, windowless rectangular chamber with dimensions of $3 \mathrm{~m}$ x $5 \mathrm{~m}$, width and length respectively. The floor and walls are of grey colour, whereas the ceiling is white, and the only existing furniture in the lab during the experiment were two computer stations, each equipped with an earset and an oculus rift viewer. One of the main advantages of this setting is the isolation from any noise and air fenestration sources.

\section{Virtual settings}

Recent studies demonstrated that the thermal sensation might be altered by using immersive virtual environments. ${ }^{44}$ However, the choice of using Virtual Reality (VR) is still convenient and handy. Therefore, two virtual settings were designed by Unity 3D program to act as a behavioural setting for the experiment. The two created virtual settings were two spaces with different physical features that include function, colour, layout and noise level. As the research design was based on factorial design, the main factor is the setting, and the minor factors are the design features including noise and colours.

The first setting is an $8 \mathrm{~m}$ x $5 \mathrm{~m}$ living space with a natural light level of (200 lux, morning sun light), coloured in white and grey and has a noise level of (35 dBA, outdoor 
live sounds). It is a single open space consisting of a sitting area and kitchenette overlooking an outdoor garden through roof-top framed window, see Figure 3 (a). The other setting is a $15 \mathrm{~m} \mathrm{x} 15 \mathrm{~m}$ public space coloured in red, yellow and orange for walls and black for ceiling along with steel frames. This space was illuminated by an artificial light level of (800 lux, indoor ceiling lamps) and a noise level (75 dBA, indoor live sounds for people and devices). It is an open work space consisting of three zones; waiting area for visitors, reception and an open administrative office which provides public services with 1.5 m x 2 m window, see Figure 3(b).

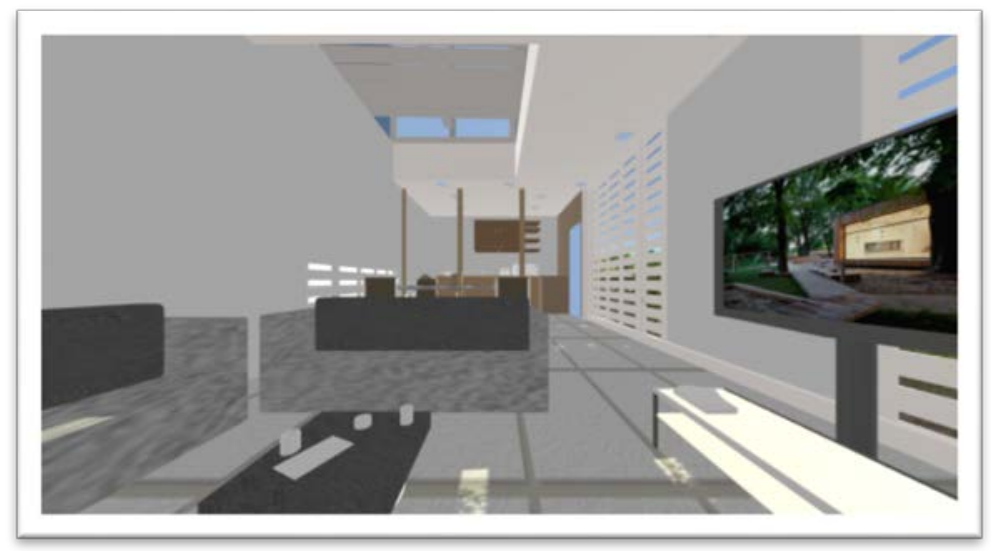

3(a) Setting I

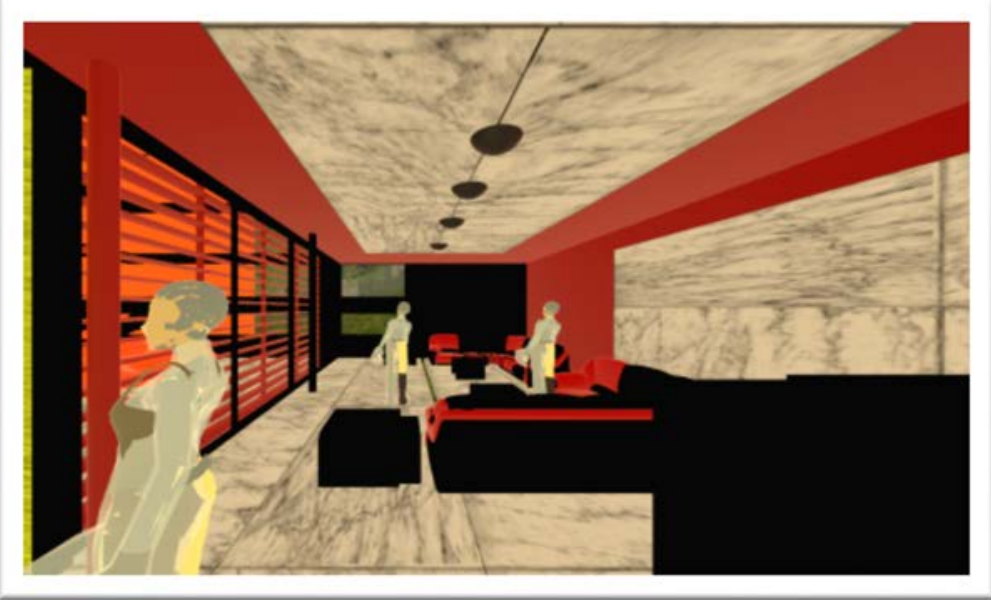

3(b) Setting II

Figure 3. The designed virtual settings. 


\section{Film clips}

Two film clips were selected to elicit two emotions (happiness and anger respectively). The length of each film clip was about 3 minutes. The selected films were chosen based on previous literature on environmental psychology and emotion eliciting criteria which validate that those clips are effective in mood elicitation. ${ }^{45}$ Additionally, those clips are not commonly known for the participants`cultural background. To elicit the happiness mode state, a film clip derived from “There is something about Mary” was used. Particularly, the cynical fighting scene between Harry and Mary’s favourite dog. The second one is derived from "My Bodyguard”, where a group of bullies attack a poor young man sitting with his friends; and they hurt him in a very provocative manner. Both film clips were screened in English accompanied by Arabic subtitles, the mother tongue of the participants. After watching the clips, a self-reported technique was used to evaluate the elicited mood that use a set of rating scales. This technique represent any set of emotions and can be used to measure combinations of emotions. ${ }^{46}$

\section{Approach}

Data were collected using a self-reported questionnaire. The questionnaire was designed to investigate the achievement of emotional elicitation and the thermal comfort perception, in which the participants have to fill out during the experiment's stages. The questionnaire included four main parts: general personal information, pre-mood test, selfreported emotional scale and thermal comfort evaluation scale.

\section{Mood tests}

The choice was made to use the PANAS- X test for this study as a pre-mood evaluation tool. This test was developed and verified by Watson and Clark. ${ }^{47}$ It includes 60 items and measures 11 specific effects: fear, sadness, guilt, hostility, shyness, fatigue, 
surprise, joviality, self-assurance, attentiveness and serenity. Thus, the PANAS-X provides either a positive effect or a negative one for mood measurement. ${ }^{47}$ This test was used to examine participants' emotions one hour before the experiment. The second one is a selfreported emotional scale; when the participant watched film-clips, the induced mood had to be evaluated by “The Positive and Negative Affect Schedule”. It is a twenty point scale ranged from (-10 very unpleasant to 10 very pleasant). ${ }^{48}$ This scale is divided into two levels; the negative sign numbers for anger and the positive sign numbers for happiness. The levels of the negative part are: $(0$ to -4$)$ is low, $(-5)$ is medium and (-6 to -10$)$ is high. While the levels of the positive part are: (0 to 4$)$ is low, (5) is medium and from (6 to 10$)$ is high.

\section{ASHARE 7-point test of TC}

This scale was developed by the American National Standards Institute (ANSI) based on Fanger`s PMV index. ${ }^{49}$ Also, it has been widely used to measure subjective responses of subjects in thermal comfort studies especially those in the multivariate approach. ${ }^{50}$ The participants rate their TC on a scale with values ranging from -3 , indicating cold, over 0 , indicating neutral, to +3 , indicating hot.

\section{Experiment procedure}

The experiment process was designed based on a full $3 \times 3 \times 2$ factorial design to build two psycho-thermal models (happiness and anger) by applying Fanger's ${ }^{6}$ " thermally controlled chamber methods". The experiment was completed in three stages that have three different thermal conditions $\left(15^{\circ} \mathrm{C}, 21^{\circ} \mathrm{C}\right.$, and $\left.27^{\circ} \mathrm{C}\right)$ that have a one-week gap in between, see Figures 4 and 5. The experiment was conducted through the following procedure: 


\section{a. Preparing the lab to the pre-set temperature}

Before the participants entered the lab, the inside temperature was set using a split unit air conditioner at the demanded operative temperature (e.g. $\left.15^{\circ} \mathrm{C}\right)$, the designated temperature of this experiment. A digital thermostat with $0.5^{\circ} \mathrm{C}$ accuracy was used to measure the inside temperature of the lab and ensure its consistency along the whole time of the experiment.

\section{b. Pre-evaluation emotional state self-report}

The participants were asked to sit down in the transitional space of the lab, then each participant was provided with a copy of PANAS-X scale and was asked to complete the first part of the questionnaire to report their emotional state prior to the experiment. This stage took approximately ten minutes.

\section{c. Emotion eliciting stage}

At this stage, each participant was asked to watch the emotion-eliciting film-clip to trigger the required mood state. Subsequently, the participant was asked to retake the Positive and Negative Affect Schedule test ${ }^{47}$ to report their overall mood on -10 to 10 scale to verify whether or not their mood had dissipated.

Each participant was asked to take 30 minutes break after finishing the first part of the experiment. The break duration was based on experts' consultation about the needed

time for a human being to depart a certain stimulated mood state. The consultants indicated that the order of elicited moods will not significantly impact the results as long as a certain mood state was neutralized appropriately. In addition to the consultation, a test was made on 10 participants, half of them experienced the anger state initially and the other half 
experienced the happy state first, with a 30-minute interval time. The reported results did not show any impact of the order of elicited moods.

\section{d. Immersive environments stage}

Directly after finishing the previous step, the participants were immersed in the two virtual environments. Those environments were projected on a computer screen, then each participant was asked to freely explore each environment for approximately 10 minutes; through wearing Oculus glasses and navigating using the mouse and keyboard keys in addition to turning their head around. This time period of 10 - 20 minutes was adequate for immersive experiments to avoid the side psych- physio effects. ${ }^{51,52}$ The two environments were presented interchangeably to the participants, i.e. half the participants experienced the domestic environment first, whereas the other half experienced the public space environment first. After fully navigating each environment, the participants were asked to complete the third part of the questionnaire which is the ASHRAE-7 points scale to determine participant's thermal satisfaction votes, as well as give an estimation of the temperature of each navigated environment. 


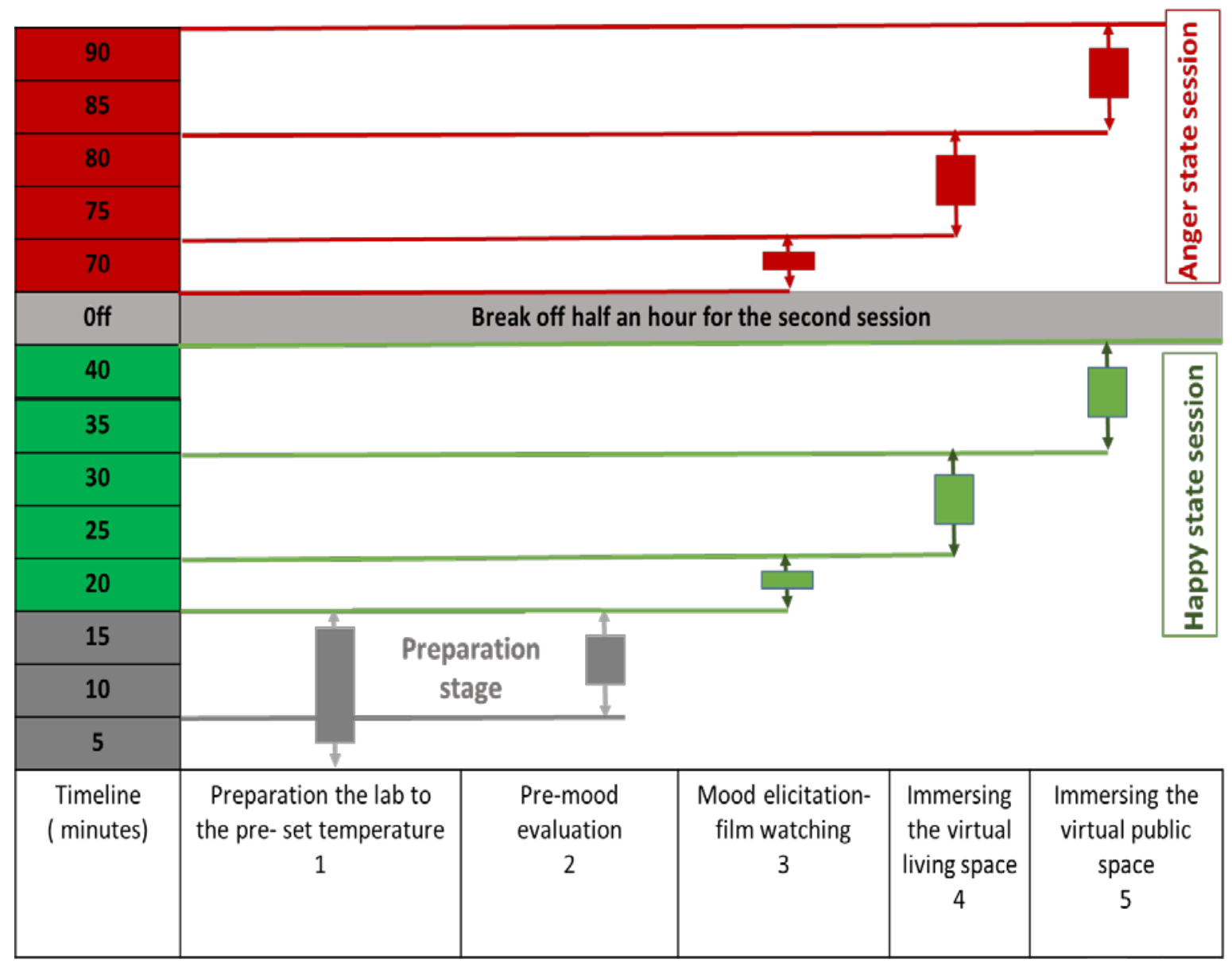

Figure 4. Timeline at each mood state during one thermal level. 


\section{Experiment Procedure}

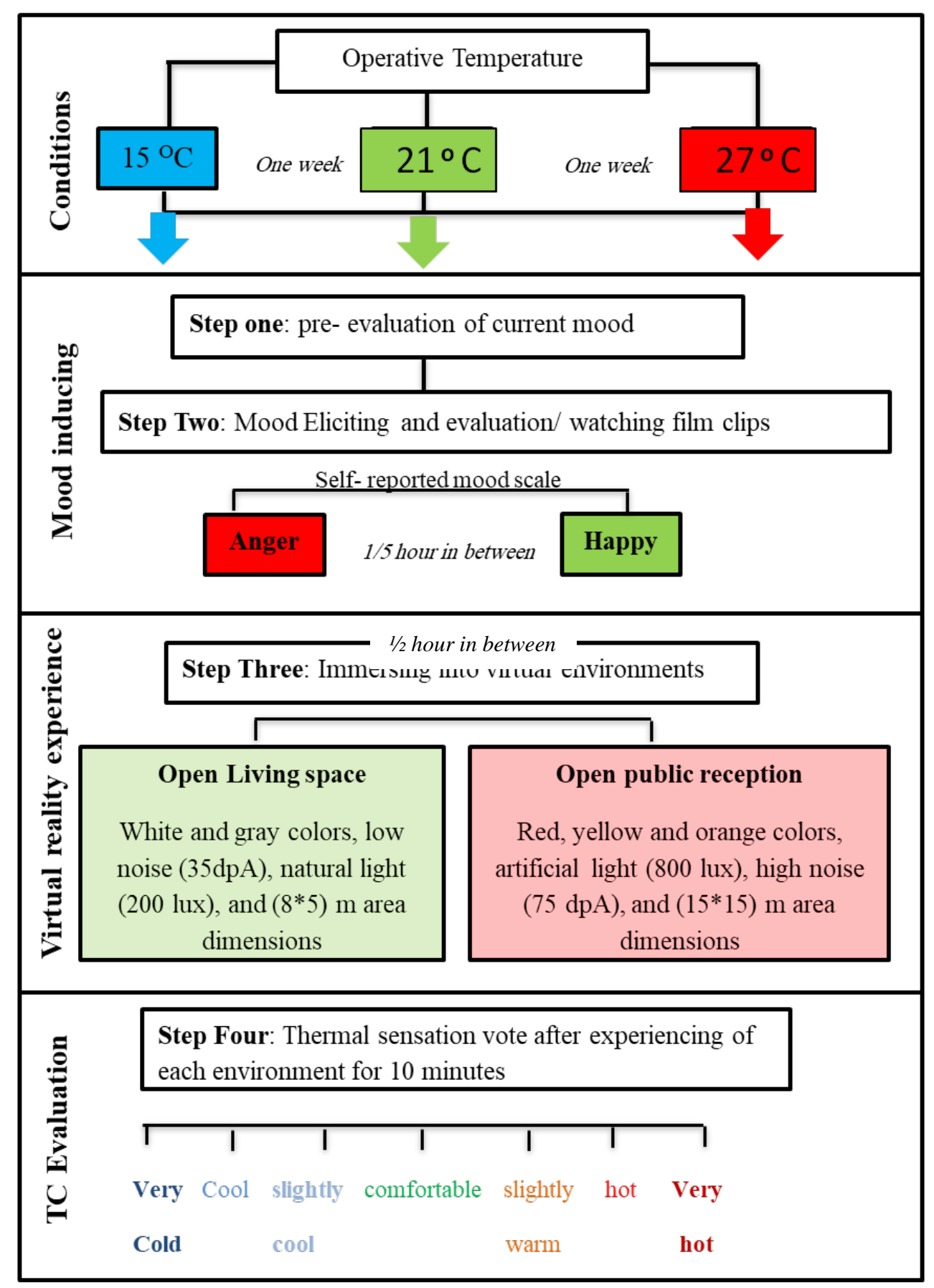

Figure 5. Expirement process. 


\section{Analysis and results}

A simple statistical General Linear Model (GLM) was used to analyse the collected data. It examines the variances of the main and interaction effects of factorial experiments variables through SPSS program. ${ }^{53}$ Two psych-thermal models were examined: happiness and anger models to specify the significant main effects in reference to the independent variables, as well as the interaction effects in reference to the interconnection between the independent variables. Descriptive and detailed statistics for major findings of those models are shown in Table 2.

Table 2. The major descriptive statics of psych-thermal models.

\begin{tabular}{l|ccc|cc}
\hline $\begin{array}{l}\text { Psych-thermal } \\
\text { model }\end{array}$ & $\begin{array}{c}\text { Operative } \\
\text { Temperature }\end{array}$ & Mood level & Setting & ${\text { Mean }{ }^{\text {a }}}$ & Standard Deviation \\
\hline Anger model & 21 & Medium & 1 & 0.05 & 0.91 \\
& 15 & high & 1 & -0.38 & 0.89 \\
& 27 & Low & 2 & 1.6 & 1.07 \\
Happy model & 21 & high & 1 & 0.00 & 0.45 \\
& 15 & high & 1 & -0.65 & 0.7 \\
& 27 & high & 2 & 1.14 & 1.6 \\
\hline
\end{tabular}

a: Mean of response TC

ASHARE 7-point scale designates the value 0 as the optimum comfort level; values above 0 indicate a warm feeling and values below 0 indicate a cold one. The results of TC average indicate that in both models, the thermal feeling is highly correlated with operative temperature, while its relationship with happiness level is constant; its relation with anger level is opposite. Also setting one correlated with low thermal feeling and setting two correlated with high thermal feeling.

In discussing the results of mood elicitation, $41.7 \%$ of the participants reached a high level of anger, then the medium and low levels respectively. While during happiness 
elicitation, $42 \%$ of the participants reached a medium happy level then the high and low levels respectively, see Figure 6. The results indicate that anger elicitation is more effective than the happiness one. The elicitation level depends on several issues including the film content, arousal level and individual differences.

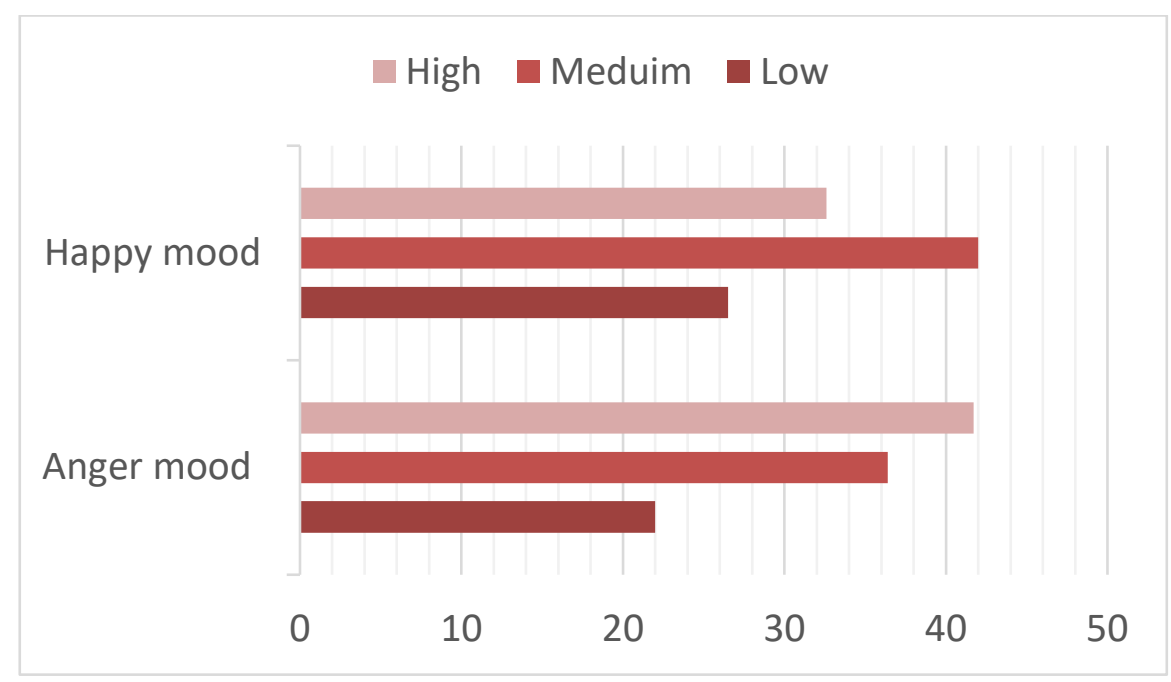

Figure 6. The post-evaluation mood scale test percentages during the two mood states.

\section{The psychological models of thermal sensation votes}

Two different perceptual thermal sensation vote models were designed based on happiness and anger mood types. There are two types of errors in the models: due to the sampling and due to the respondents which is natural in all modeling systems. However, the significant level is the determinant of the influential factors affecting the happiness and anger mood models. By comparing the two models, the result of perceived thermal sensation shows that at anger mood. The thermal comfort Level (TCL) evaluation had a normal distribution, and the highest evaluation percentage at the neutral level was about 57\%. On the other hand, at happy mood, TCL distribution was shifted to the warm part, and the highest evaluation percentage at a slightly warm level was about $32 \%$. The extreme thermal votes were below 5\% which is low rated. The slightly cool vote is highly rated in 
the case of anger state more than the happy one which is about $22 \%$. On the other hand, $15 \%$ of participants felt slightly warm at the anger state which is less than the happy one which was about $32 \%$. Finally, 21\% of respondents felt hot in the happy state whereas this percentage is higher than that at the anger state, see Figure 7.

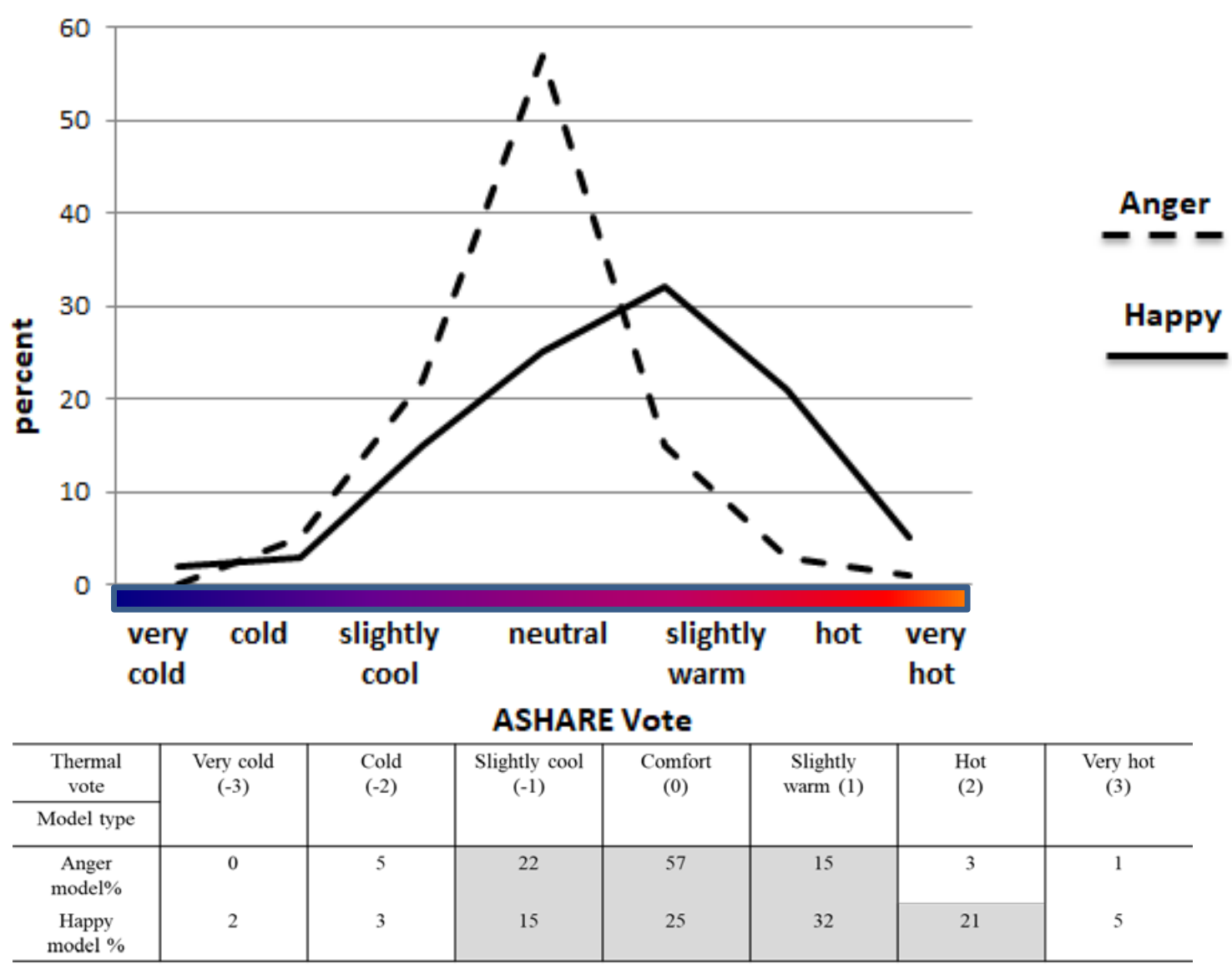

Figure 7. Destribution of Thermal Sensation Vote for two psych-thermal models.

\section{The main interaction effects of psych-thermal models}

By comparing the percentage of significant main effects variables (p- value less than 0.05), the operative temperature is more effective in anger model. However, the virtual setting is highly effective at the happy model, and the mood level does not seem significant at all. However, at the anger model the participant's evaluation of their thermal sensation 
regarding the temperature is higher. These findings indicate that the negative mood increases the perception of warm feeling while the positive one decreases it.

A positive relationship between settings and TCL in both psych-thermal models was found. Actually, the virtual settings were designed to test specific parameters. In general, the first setting has a neutral conditions whereas the thermal sensation is cold. However the second setting has highly extreme conditions, so the thermal sensation is slightly warm; but the subjects voted the coldest feeling at (environment one/ happy state) and the warmest feeling at (environment two/ anger state), see Figure 8.

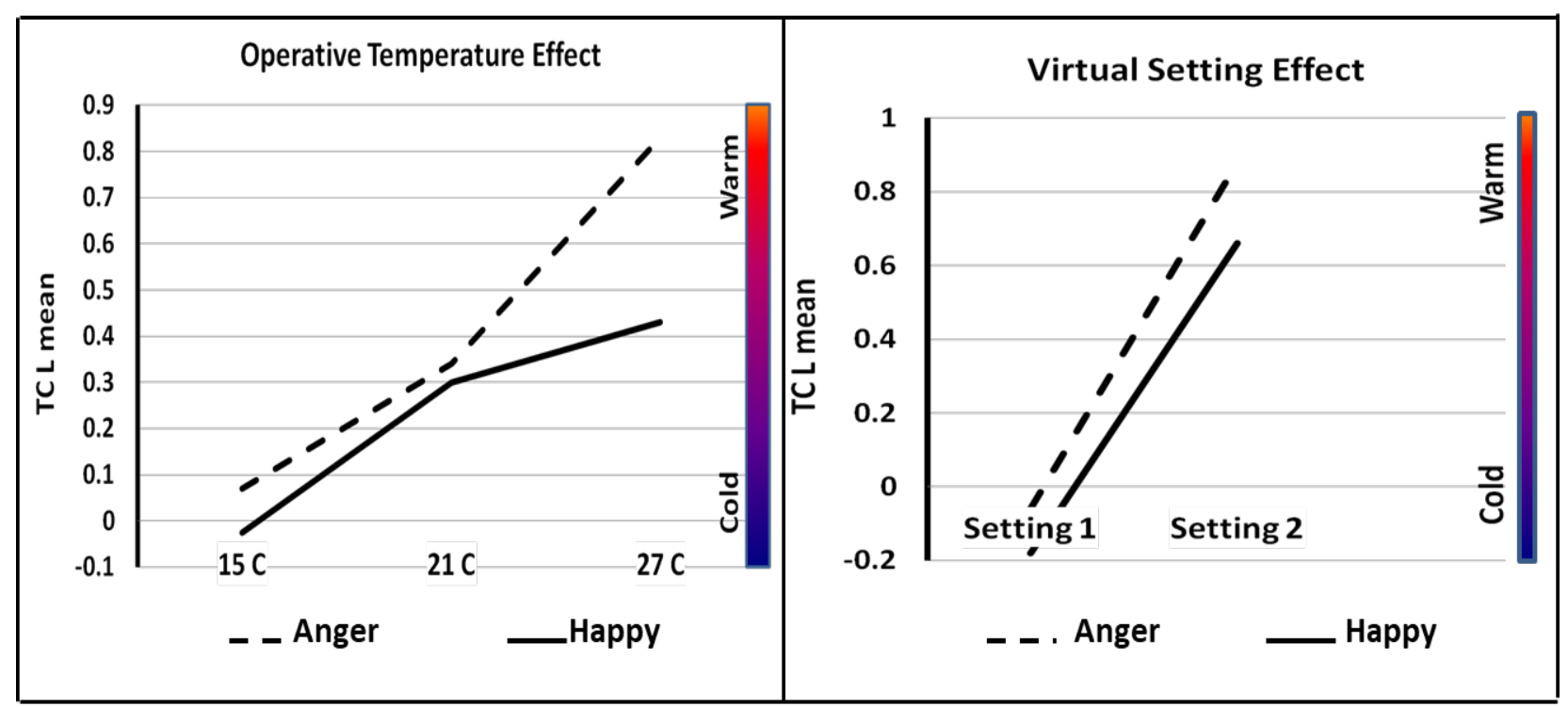

\begin{tabular}{|c|c|c|c|c|}
\hline Variable & \multicolumn{2}{|c|}{ Anger } & \multicolumn{2}{|c|}{ Happy } \\
\hline Operative temperature (To) & 0.000 & $7 \%$ & 0.005 & $4 \%$ \\
\hline Virtual setting (VS) & 0.000 & $16 \%$ & 0.000 & $18 \%$ \\
\hline Model level (ML) & N.S & $0.5 \%$ & N.S & $0.3 \%$ \\
\hline
\end{tabular}

Alpha $=0.1$, N.S $=$ Not Significant

Figure 8. The main effect of significant variables in each model.

The physical attributes of the built environment vs thermal sensation

A set of environmental characteristics are tested including colour (warm, cold), noise (neutral, high), and light (artificial, natural). On environment one, the living space, Figures 
9 and 10 show a significant influence of colour on thermal evaluation. The white colour stimulates a slightly comfortable feeling $(-0.08)$ at the happy mood state, and it was not an active factor in the anger case. The noise parameter decreased TCL 0.96 degree to be -0.2 , slightly cool at the happy state, and increased the TCL 1.2 degrees to be about 0.14 , around slightly warm at the anger state. However, natural light motivates the warm sensation to be about 1 at anger mood state, while it caused the highest slightly warm sensation 0.75 in comparison with the effect of each other properties at the happy state. Finally, during TCL evaluation in anger state, the participants altered initially to environment one as a whole, then they took each parameter alone. Hence, during the happy state, they altered to each single parameter alone. These findings indicate that cognition process during anger mood is fragmented and disordered, while it is more organized and clear at the happy state.

In the second environment, public workspace, the pairwise comparison of the environment characterstics shows that the anger mood did not cause a significant difference or any thermal effect at all. While during the happy mood, warm colours increases the sensation vote in comparison with all properties together which decreased the participants' thermal sensation to be slightly comfortable. The noise parameter also increased the participant thermal sensation vote in comparison with all properties together to be slightly warm. Eventually, the artificial light has neutral thermal effect. Therefore, during the happy state, environment properties separately increased the warm effect, but the interaction between them decreased thermal sensation. 


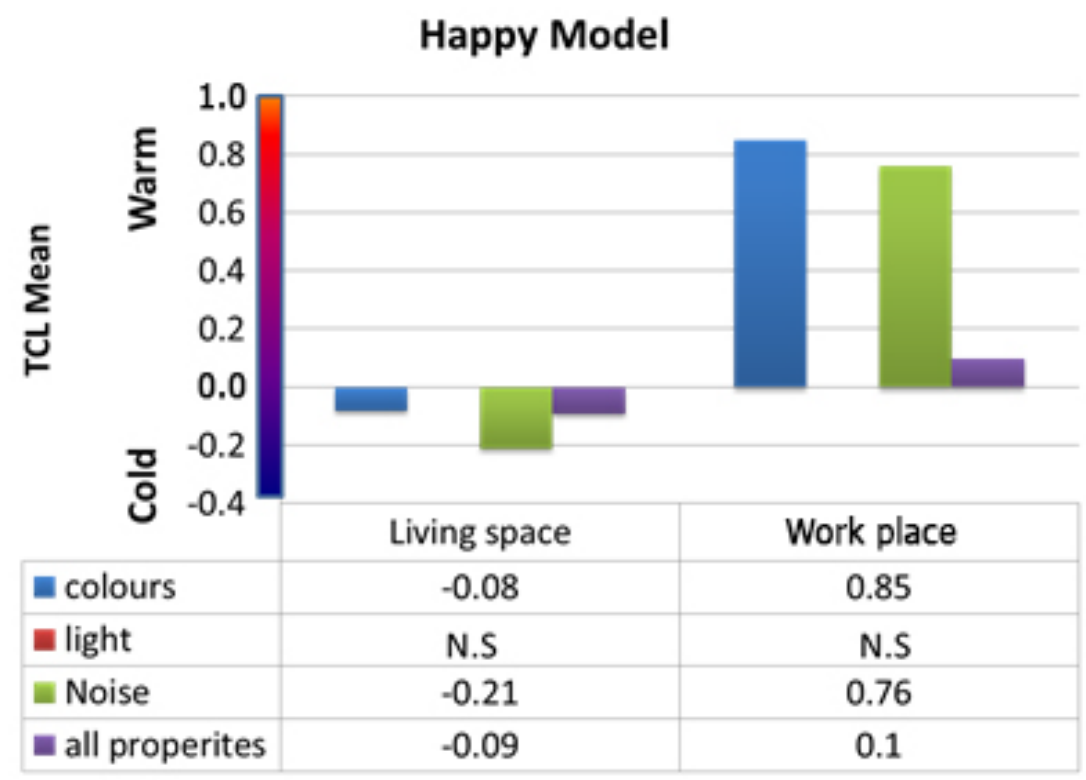

Figure 9. The main effect of the physical artibutes on TCL in Happy psych- thermal model

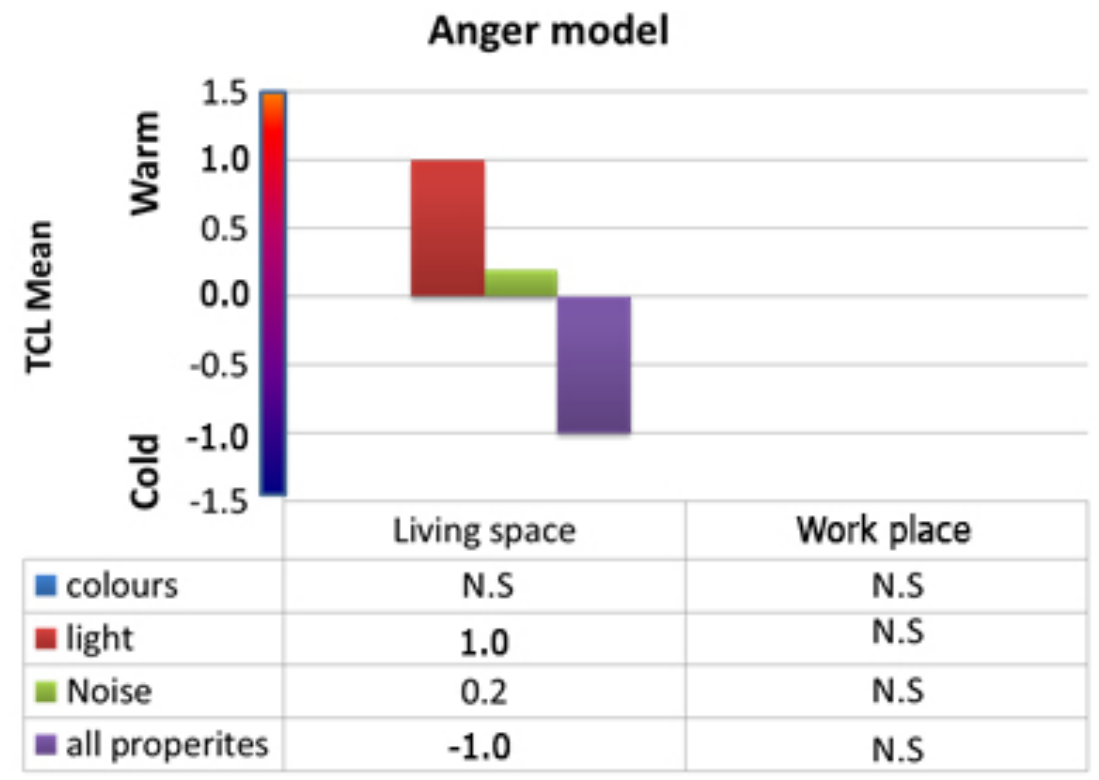

Figure 10. The main effect of the physical artibutes on TCL in Anger psych-thermal model.

Although the independent variable may not be separately affective, it interacts with other independent variable(s) in an effective way. By comparing interaction effects for the psych-thermal models, the results showed that Mood Level interaction with each Operative Temperature in the anger model, and Virtual Setting in the happy one has a significant 
impact on TCL. However, the participants within negative mood state were affected by the temperature while in positive mood state they were affected by the setting design properties.

Through analysing the interaction of mood level and operative tempreature during the anger state, the results shown in Figure 11 reveal that the extreme mood states interact clearly with the thermal condition. For example, low anger state is close to natural state, therefore TCL evaluation will be affected more by real thermal conditions. The comfortable case was during the interaction between $15^{\circ} \mathrm{C}$ and low anger. The warmest feeling was during the interaction between $30^{\circ} \mathrm{C}$ and high anger level. Finally, the coldest feeling was during the interaction between $15^{\circ} \mathrm{C}$ and medium anger. 

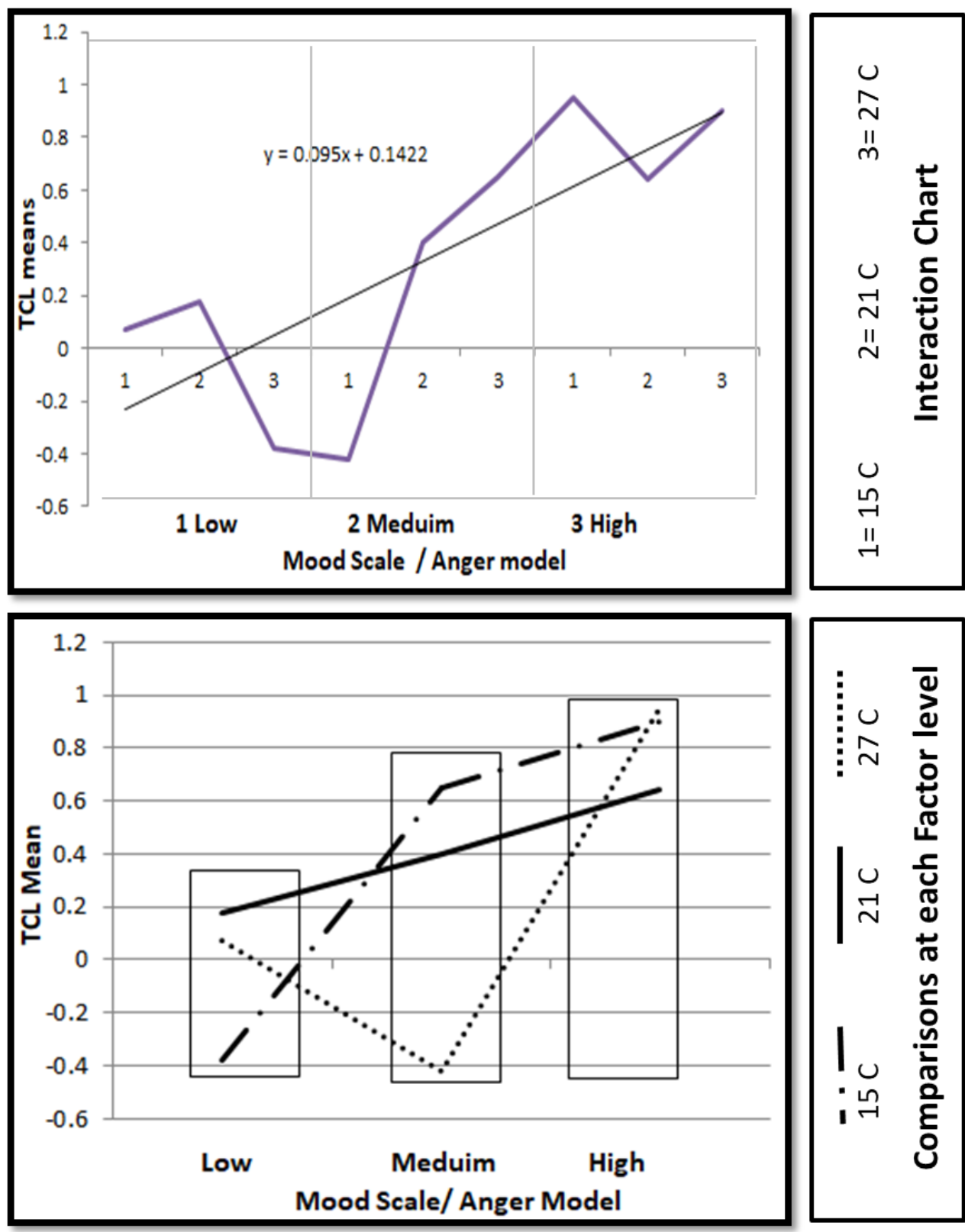

Figure 11. The interaction effect of mood level and operative temperature on TCL.

When examining the interaction of mood level and virtual setting during happy state, the results reaveal that when participants experienced environment two (high extreme conditions), they felt slightly warm, see Figure 12, while during their time at environment one (neutral conditions), they felt cooler. Therefore, the higher happy levels and highquality virtual setting increased TCL. In other words, TCL average of low and high mood levels interaction with virtual settings is higher than medium-and virtual setting interaction. 
Approximate comfortable case was in the interaction between medium happy scale and the first virtual space. The warmest case was at the high level of happiness across the second virtual space, and the coldest case was in the interaction between high happy state and first virtual space.

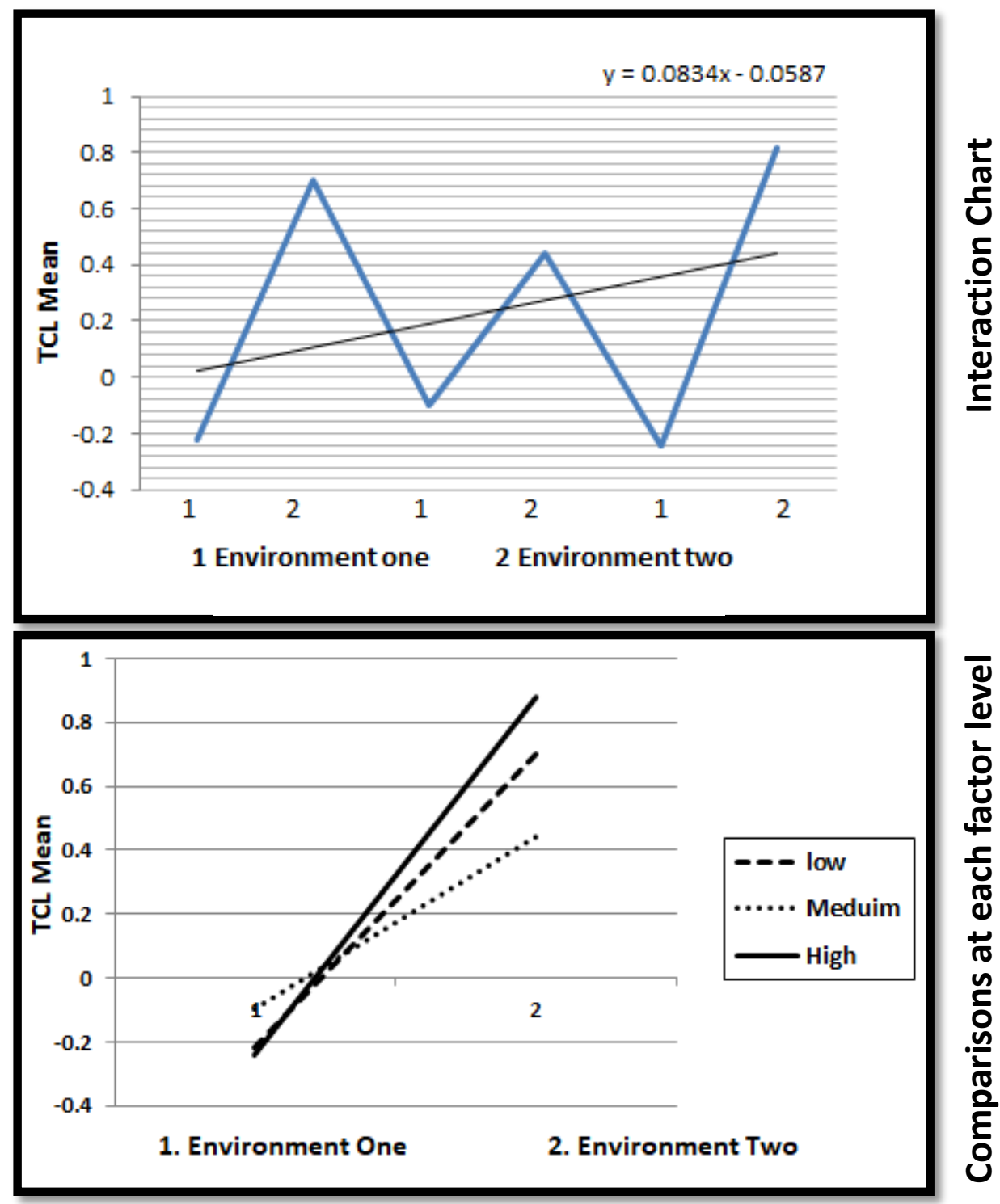

Figure 12. The interaction effect of mood level and virtual setting on TCL at each level.

Finally, the negative mood state was more influential than the positive one on the TC level. However, the interaction effect results in each psych-thermal model clarified that 
mood interaction at extreme levels, either low or high, differed from medium level; whereas the mood filtration of thermal comfort perception is pronounced in extreme mood levels than medium level. This result indicates that perceptual thermal feeling needs specified mood levels to be evaluated and judged.

\section{Anger model vs happy model}

Comparing the most effective model shows that anger model is highly significant and the strength of this model is about $30 \%(r=0.3)$, which indicates the moderate strength of the model hypothesis. On the other hand, the results of the happy model show that this model is significant but the effect of this model is less than anger model, it is about 23\% ( $r=0.23)$, which is slightly weak. This was achieved by the variances of the main effects including (A) operative temperature; (B) anger/ happy scale; and (C) virtual setting and the interactions between them. Equation (1) is the statistical model used to evaluate TCL during anger and happy state. Equation (1) includes the individual effects as well as their interactions:

$\mathrm{TCL}=\beta_{0}+\beta_{1}\left(\mathrm{~A}_{\mathrm{i}}\right)+\beta_{2}\left(\mathrm{~B}_{\mathrm{i}}\right)+\beta_{3}\left(\mathrm{C}_{\mathrm{i}}\right)+\beta_{4}\left(\mathrm{~A}_{\mathrm{i}} \mathrm{xB}\right)+\beta_{5}\left(\mathrm{~A}_{\mathrm{i}} \mathrm{xC}\right)+\beta_{6}\left(\mathrm{~B}_{\mathrm{i}} \mathrm{xC} \mathrm{C}_{\mathrm{i}}\right)+\beta_{7}\left(\mathrm{~A}_{\mathrm{i}} \mathrm{xB}_{\mathrm{i}} \mathrm{xC} \mathrm{C}_{\mathrm{i}}\right)+$ error $_{i}$

( $\mathrm{i}$ = parameter no, 1, 2, 3, n, A = OT, B = ML, C = VS, TCL = Thermal Comfort Level)

The humans' mood state in solitude does not provide an explanation to the perceived thermal sensation, as it is related to other physical factors explained by Fanger. ${ }^{6}$ However, as the results indicate, the thermal perception was highly subjective under the influence of certain mood states. In general, the negative mood state influences the perceived thermal sensation while the positive mood, if not neutral in the process, enriches the feeling of the actual operative temperature. A happy mood state will give a thermally comfortable feeling in a standard comfort zone situation regardless of the physical attributes of the setting. According to Forgas, ${ }^{54}$ the positive mood tends to improve the cognition and memory more 
than the negative mood, therefore humans evaluate their thermal sensation according to the actual thermal ambiance.

The results revealed that through anger state the higher temperature increases TCL rating above actual temperature. Whereas, the interaction effect of higher temperature and higher anger level will increase warm sensation and vice versa. This indicates that humans' thermal perception is more sensitive to temperature change especially during bad moods. Batra and Garg ${ }^{32}$ support the previous concept through their findings, temperature plays an important role in humans’ memory and perception. In addition, Denissen et al. ${ }^{55}$ state that negative mood is highly correlated with temperature.

On the other hand, during happy state, TCL rating is compatible with actual temperature. Therefore, in the case of a positive mood, the ambient temperature is significantly important. Referring to Forgas, ${ }^{54}$ this finding indicates that psychologically, the positive mood has a more pronounced effect on evaluations and memory, therefore humans evaluate their thermal sensation according to how they actually perceive the surrounded environmental condition. In other words, when humans feel highly happy in neutral and quiet environmental setting, they are likely to be more precise in evaluating their thermal sensation.

\section{Discussion}

The humans' mood state in solitude does not provide an explanation to the perceived thermal sensation, as it is paired with other physical factors explained by Fanger. The physical design features of the virtual space affect human evaluation of the thermal environment. Additionally, colour and noise affect the mood state, which influences the cognitive process for humans and acts as a layer to filter evaluations and perceptions. 
Therefore, positive mood contributes well to improve the sense of heat, and in contrast, negative mood disrupts the cognitive process, so the sense of heat becomes turbulent or sometimes illogical.

From the results of those psych-thermal models: happiness and anger models, there is a variation on Interaction Effect level. This variation may be related to: first, the individual differences of TC perception which has a negative impact on TCL. ${ }^{56}$ Second, the personality traits differences for participants. Some traits influence negatively - such as neuroticism - on TCL while others influence positively. ${ }^{57}$ Third, differences in emotional appraisal level based on surrounding conditions. Sinclair et al. ${ }^{35}$ argued that temperature is possibly mediated through humans' mood appraisal level. This means that the subjective approach of TC does not have constant results due to the subjective parameters and techniques. Therefore, such approach needs a specific evaluation technique by including extreme categories of cold and hot sensation to be compatible with individual differences factors.

In terms of virtual settings, in both psycho-thermal models, natural conditions of virtual environments registered the highest comfort percentage (living space), especially in an angry mood. In private settings, the participant is provided with high control over the setting`s features which enhances the mood. ${ }^{58}$ In addition, the mood sensitivity of people who are less satisfied with the environmental features will be affected. ${ }^{59}$ This finding correlates with Russell and Snodgrass' argument that the sensory inputs of the surrounded environment are the main source that can formulate individual`s moods. ${ }^{60}$

In discussing the effect of the physical features of the virtual setting, the research demonstrated that white colours and 35 dBA noise level are correlated with anger mood. On the other hand, warm colours and 75 dBA noise level were more active and influential in the thermal experience. In the positive mood, they motivate warm feelings, however, 
they motivate hot feelings at the anger case. These findings resonate with the findings of Pellerin and Candas ${ }^{61}$ that noise may alter thermal satisfaction in warm conditions. Also, these findings support the claim that aggressive mood is reduced when the participant is in a dominated cool-colour environment. ${ }^{62}$ Furthermore, warm colours have the tendency to increase the arousal more than cool colours which will directly impact the human perception of various aspects of space, including one's thermal perception. ${ }^{63}$ This is in agreement with previous research which suggests that some colours evoke psychological reactions through signals such as warmth ${ }^{64}$; and with Fanger`s ${ }^{6}$ findings that warm colours alter the human thermal perception more than cold colours.

This research hypothesized that humans' mood directly influences thermal sensation. However, the results indicated that the mood works as a mediator of our thermal evaluations by sharing either the extreme thermal conditions in its negative state, or the design attributes in the positive state, so it is a null hypothesis. According to physical design attributes, the research highlighted their role as moderators that have the ability to enhance humans’ mood and their thermal sensation. Cold colours and quiet sounds; especially in living spaces, improved human evaluation of their thermal experience. On the other hand, warm colours and noisy environments motivated the human thermal discomfort in the pleasant mood. Therefore, moods can be considered as significant subjective and effective media through which people evaluate their TC level.

In terms of light source, natural vs. artificial; natural light particularly in happy state, motivated the warm sensation in comparison with other parameters. These results indicate that when the light luminance increases, the emotional actions are revealed, which in turn affects human thermal sensation. This result is incongruent with a study finding that argued that bright light is not an effective intervention for enhancing feelings of well-being. ${ }^{65,} 66$ Also natural lighting, especially in offices, produces sensations of satisfaction and warmth. 
Even in an air-conditioned and thermally comfortable room, people are still attracted to sunlight. ${ }^{58}$

In general, the findings regarding the design attributes support earlier research which demonstrated that the relationship between humans' mental action and the environmental aspects is one of the main factors that affect people's judgment on spaces. ${ }^{17}$ Extreme conditions of any space formulate the way which human perceive temperature. Therefore, thermal perception is a result of selective interpretation of humans' minds. On the other hand, the neutral conditions have opposite effect. Positive and negative moods have residual effects on human cognition in general. In fact, positive and negative moods influence a wide range of judgments, including thermal perception and evaluation. Whereas negative moods reduce people’s ability to attain any performance and increase human de-motivation. In addition, extreme conditions increase with the negative state, which in turn increases the confusion of thermal evaluation pattern. This finding is contradicting what Kobrick and Johnson ${ }^{67}$ reported in terms of the impact of extreme conditions of any environment on psychological prior to physiological factors. ${ }^{67}$

\section{Conclusions}

This research examined the effect of various mood states on the human perception and evaluation of the thermal environment under three operative temperatures in two virtual settings. The study findings demonstrated that mood states have a strong effect on our thermal perception and evaluation. Mood states are also connected with the design attributes of the environment. Therefore, humans' judgment on their thermal comfort is not a constant objective process but a subjective dynamic mental reaction.

In conclusion, this research contributes to further understandings of the process of evaluating the humans' thermal sensation as mediated by mood state. This study expands 
research on indoor environment quality and develops TC evaluation strategies through involving subjective psychological parameters. Encompassing such knowledge with TC models presumably will improve the operation of these models and their ability to explain the individual differences more efficiently. One of the limitations of this study is that other non-thermal aspects such as cultural and personality differences were not considered. Future research should reflect on cultural and personality differences to explain their impact on how humans perceive their thermal sensation. Future studies can develop research methods in TC studies to improve the quality of existing subjective assessment and evaluation tools, by involving additional subjective variables related to personal and non-physical parameters. This research also informs design practice by highlighting flexible design elements (e.g. colours, furniture, light, visual and acoustic comfort) to control occupants' mood state and improve their TC. In addition, this study is supported by earlier research on VR application as a useful tool to examine the thermal quality of architectural designs at the design stage; and to predict the occupants' TC with design elements.

\section{Authors' contribution}

All authors contributed equally in the preparation of this article.

\section{Declaration of conflicting interests}

The authors declared no potential conflicts of interest with respect to the research, authorship, and/or publication of this article.

\section{Funding}

This research was not funded by any financial resource. 


\section{References}

1. Maiti R. PMV model is insufficient to capture subjective thermal response from Indians. International Journal of Industrial Ergonomics 2014; 44: 349-361.

2. Parsons K. Human thermal environments: the effects of hot, moderate, and cold environments on human health, comfort, and performance. Boca Raton, FL, USA: CRC press, 2014.

3. Heijs W and Stringer P. Research on residential thermal comfort: some contributions from environmental psychology. Journal of environmental psychology 1988; 8: 235-247.

4. Olgyay V. Bioclimatic approach to architecture. BRAB conference report. National Research Council Washington, DC, 1953.

5. Auliciems A and Szokolay SV. Thermal comfort. Brisbane: PLEA, 1997.

6. Fanger PO. Thermal comfort Analysis and applications in environmental engineering. Copenhagen: Danish Technical Press, 1970.

7. Han J, Zhang G, Zhang QQ, Zhang J, Liu J, Tian L, Zheng C, Hao J, Lin J, Liu Y and Moschandreas DJ. Field study on occupants' thermal comfort and residential thermal environment in a hot-humid climate of China. Building and Environment 2007; 42: 4043-4050.

8. Wang Z. A field study of the thermal comfort in residential buildings in Harbin. Building and Environment 2006; 41: 1034-1039.

9. Griffit W and Veitch R. Hot and crowded: Influence of population density and temperature on interpersonal affective behavior. Journal of Personality and Social Psychology 1971; 17: 92.

10. Howell WC and Kennedy PA. Field validation of the Fanger thermal comfort model. Human Factors 1979; 21: 229-239.

11. ISO 7730:2005. Ergonomics of the Thermal Environment: Analytical Determination and Interpretation of Thermal Comfort Using Calculation of the PMV and PPD Indices and Local Thermal Comfort Criteria. International Organization for Standardization, Geneva, 2005.

12. Nikolopoulou M, Baker N and Steemers K. Thermal comfort in outdoor urban spaces: understanding the human parameter. Solar energy 2001; 70: 227-235.

13. Andersen RV, Toftum J, Andersen KK and Olesen BW. Survey of occupant behaviour and control of indoor environment in Danish dwellings. Energy and Buildings 2009; 41: 11-16.

14. Foda E and Sirén K. Evaluating the thermal comfort performance of heating systems using a thermal manikin with human thermoregulatory control. Indoor and Built Environment 2016; 25: 191-202.

15. Azizpour F, Moghimi S, Lim CH, Mat S, Salleh E and Sopian K. A thermal comfort investigation of a facility department of a hospital in hot-humid climate: correlation between objective and subjective measurements. Indoor and Built Environment 2012; 22(5): 836-845.

16. Gross JJ and Barrett LF. Emotion generation and emotion regulation: One or two depends on your point of view. Emotion review 2011; 3: 8-16.

17. Stemmler G and Wacker J. Personality, emotion, and individual differences in physiological responses. Biological psychology 2010; 84: 541-551.

18. Schiffman HR. Sensation and perception: An integrated approach. Oxford, England: John Wiley \& Sons, 1990.

19. Lin IY. Evaluating a servicescape: the effect of cognition and emotion. International Journal of Hospitality Management 2004; 23: 163-178.

20. Smith CA and Lazarus RS. Emotion and adaptation. In : Pervin L Handbook of personality: Theory and research, New York: Guilford Press, 1990: 609-637.

21. Keltner D, Ellsworth PC and Edwards K. Beyond simple pessimism: effects of sadness and anger on social perception. Journal of personality and social psychology 1993; 64: 740.

22. Lane AM, Terry PC, Stevens MJ, Barney S and Dinsdale SL. Mood responses to athletic performance in extreme environments. Journal of sports sciences 2004; 22: 886-897.

23. Veitch R and Arkkelin D. Environmental psychology: An interdisciplinary perspective. Pearson College Division, London, 1995.

24. Yildirim K, Hidayetoglu ML and Capanoglu A. Effects of interior colors on mood and preference: comparisons of two living rooms. Perceptual and motor skills 2011; 112: 509-524.

25. Wexner LB. The degree to which colors (hues) are associated with mood-tones. Journal of applied psychology 1954; 38: 432.

26. Boyatzis CJ and Varghese R. Children's emotional associations with colors. The Journal of genetic psychology 1994; 155: 77-85.

27. Baniya RR, Tetri E, Virtanen J and Halonen L. The effect of correlated colour temperature of lighting on thermal sensation and thermal comfort in a simulated indoor workplace. Indoor and Built Environment 2018; 27: 308-316. 
28. Gou Z, Lau SS-Y and Qian F. Comparison of mood and task performance in naturally-lit and artificially-lit environments. Indoor and Built Environment 2015; 24: 27-36.

29. Sun C, Lian Z and Lan L. Work performance in relation to lighting environment in office buildings. Indoor and Built Environment 2018: $1420326 X 18820089$.

30. Muller MD, Muller SM, Kim C-H, Ryan EJ, Gunstad J and Glickman EL. Mood and selective attention in the cold: the effect of interval versus continuous exercise. European journal of applied physiology 2011; 111: 1321-1328.

31. Alden AL, Dale JA and DeGood DE. Interactive effects of the affect quality and directional focus of mental imagery on pain analgesia. Applied Psychophysiology and Biofeedback 2001; 26: 117-126.

32. Batra P and Garg R. Effect of temperature on memory. Journal of the Indian Academy of Applied Psychology 2005; 31: 43-48.

33. Taylor HL and Orlansky J. The effects of wearing protective chemical warfare combat clothing on human performance. Institute For Defense Analyses, Alexandria, Va, 1991.

34. Nadel E, Pandolf K, Roberts M and Stolwijk J. Mechanisms of thermal acclimation to exercise and heat. Journal of Applied Physiology 1974; 37: 515-520.

35. Sinclair RC, Mark MM and Clore GL. Mood-related persuasion depends on (mis) attributions. Social Cognition 1994; 12: 309-326.

36.Zhong C-B and Leonardelli GJ. Cold and lonely: Does social exclusion literally feel cold? Psychological Science 2008; 19: 838-842.

37. Song W, Liu L, Tian Y, Sun G, Fong S and Cho K. A 3D localisation method in indoor environments for virtual reality applications. Human-centric Computing and Information Sciences 2017; 7: 39.

38. Iachini T, Coello Y, Frassinetti F and Ruggiero G. Body space in social interactions: a comparison of reaching and comfort distance in immersive virtual reality. PloS one 2014; 9: e111511.

39. Pan $X$ and Hamilton AFdC. Why and how to use virtual reality to study human social interaction: The challenges of exploring a new research landscape. British Journal of Psychology 2018; 109: 395-417.

40. Miller KJ, Adair BS, Pearce AJ, Said CM, Ozanne E and Morris MM. Effectiveness and feasibility of virtual reality and gaming system use at home by older adults for enabling physical activity to improve health-related domains: a systematic review. Age and ageing 2013; 43: 188-195.

41. Feng Z, González VA, Amor R, Lovreglio R and Cabrera-Guerrero G. Immersive virtual reality serious games for evacuation training and research: A systematic literature review. Computers \& Education 2018; 127: 252-266.

42. Shin J, An G, Park J-S, Baek SJ and Lee K. Application of precise indoor position tracking to immersive virtual reality with translational movement support. Multimedia tools and applications 2016; 75: 12331-50.

43. Antony J. Design of experiments for engineers and scientists. London: Elsevier, 2014.

44. Yeom D, Choi J-H and Zhu Y. Investigation of physiological differences between immersive virtual environment and indoor environment in a building. Indoor and Built Environment 2019; 28: 46-62.

45. Schaefer A, Nils F, Sanchez X and Philippot P. Assessing the effectiveness of a large database of emotioneliciting films: A new tool for emotion researchers. Cognition and Emotion 2010; 24: 1153-1172.

46. Desmet P. Measuring emotion: Development and application of an instrument to measure emotional responses to products. In: Blythe MA, Monk AF, Overbeeke K and Wright PC (Eds), Funology: From Usability to Enjoyment. New York, NY: Kluwer Academic Publishers, 2003, pp.111-123.

47. Watson D and Clark LA. The PANAS-X: Manual for the positive and negative affect schedule-expanded form. The University of Iowa, Iowa, 1999.

48. Watson D, Clark LA and Tellegen A. Development and validation of brief measures of positive and negative affect: the PANAS scales. Journal of personality and social psychology 1988; 54: 1063.

49. ASHRAE. ASHRAE standard : thermal environmental conditions for human occupancy. Atlanta, Ga.: ASHRAE, 2010.

50. Haddad S, King S, Osmond P and Heidari S. Questionnaire design to determine children's thermal sensation, preference and acceptability in the classroom. Proceedings-28th International PLEA Conference on Sustainable Architecture + Urban Design: Opportunities, Limits and Needs-Towards an Environmentally Responsible Architecture. Lima, Peru, 7-9 Nov 2012.

51. Baños RM, Botella C, Garcia-Palacios A, Villa H, Perpiñá C and Alcaniz M. Presence and reality judgment in virtual environments: a unitary construct? CyberPsychology \& Behavior 2000; 3: 327-335.

52. Regan E and Price K. The frequency of occurrence and severity of side-effects of immersion virtual reality. Aviation, Space, and Environmental Medicine 1994; 65(6): 527-530.

53. Leech N, Barrett K and Morgan GA. SPSS for intermediate statistics: Use and interpretation. Routledge, 2013.

54. Forgas JP and Bower GH. Mood effects on person-perception judgments. Journal of personality and social psychology 1987; 53: 53. 
55. Denissen JJ, Butalid L, Penke L and Van Aken MA. The effects of weather on daily mood: A multilevel approach. Emotion 2008; 8: 662.

56. Nakano J, Tanabe S-i and Kimura K-i. Differences in perception of indoor environment between Japanese and non-Japanese workers. Energy and Buildings 2002; 34: 615-621.

57. LeBlanc J, Ducharme M, Pasto L and Thompson M. Response to thermal stress and personality. Physiology \& behavior 2003; 80: 69-74.

58. Wang N and Boubekri M. Design recommendations based on cognitive, mood and preference assessments in a sunlit workspace. Lighting Research \& Technology 2011; 43: 55-72.

59. Miwa Y and Hanyu K. The effects of interior design on communication and impressions of a counselor in a counseling room. Environment and Behavior 2006; 38: 484-502.

60. Russell JA and Snodgrass J. Emotion and the environment. In: Altman SD (ed) Handbook of environmental psychology, New York: Wiley, 1987, pp.245-281.

61. Pellerin $\mathrm{N}$ and Candas V. Combined effects of temperature and noise on human discomfort. Physiology \& Behavior 2003; 78: 99-106.

62. Kwallek N, Lewis CM and Robbins AS. Effects of office interior color on workers' mood and productivity. Perceptual and Motor Skills 1988; 66: 123-128.

63. Kurt S and Osueke KK. The effects of color on the moods of college students. SAGE Open 2014; 4: 2158244014525423.

64. Courtis JK. Colour as visual rhetoric in financial reporting. Accounting Forum 2004; 28(3): 265-281.

65. Genhart MJ, Kelly KA, Coursey RD, Datiles M and Rosenthal NE. Effects of bright light on mood in normal elderly women. Psychiatry research 1993; 47: 87-97.

66. Golden RN, Gaynes BN, Ekstrom RD, Hamer, R.M., Jacobsen, F.M., Suppes, T., Wisner, K.L. and Nemeroff, C.B. The efficacy of light therapy in the treatment of mood disorders: a review and metaanalysis of the evidence. American Journal of Psychiatry 2005; 162: 656-662.

67. Kobrick J and Johnson R. Effects of hot and cold environments on military performance. In: Gla R and Mangelsdorff (eds) Hand book of military psychology.. New York: Wiley, 1991, pp.215-232. 